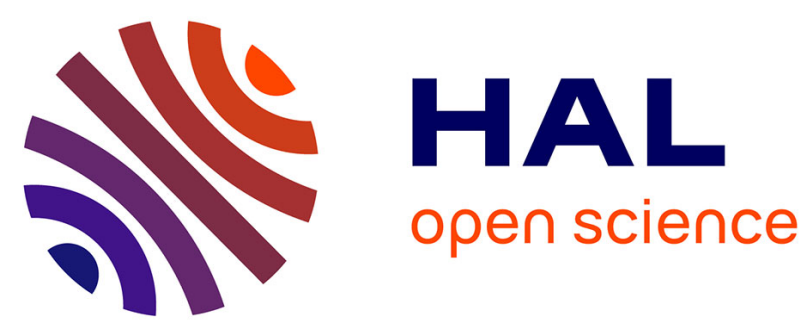

\title{
Tribology of flexible and sliding spinal implants: Development of experimental and numerical models
}

Sophie Le Cann, Julien Chaves-Jacob, Jean-Marie Rossi, Jean-Marc Linares, Patrick Chabrand

\section{- To cite this version:}

Sophie Le Cann, Julien Chaves-Jacob, Jean-Marie Rossi, Jean-Marc Linares, Patrick Chabrand. Tribology of flexible and sliding spinal implants: Development of experimental and numerical models . Journal of Biomedical Materials Research Part B: Applied Biomaterials, 2018, 106 (1), pp.104-111. 10.1002/jbm.b.33819 . hal-01691573

\section{HAL Id: hal-01691573 \\ https://hal-amu.archives-ouvertes.fr/hal-01691573}

Submitted on 14 May 2018

HAL is a multi-disciplinary open access archive for the deposit and dissemination of scientific research documents, whether they are published or not. The documents may come from teaching and research institutions in France or abroad, or from public or private research centers.
L'archive ouverte pluridisciplinaire HAL, est destinée au dépôt et à la diffusion de documents scientifiques de niveau recherche, publiés ou non, émanant des établissements d'enseignement et de recherche français ou étrangers, des laboratoires publics ou privés. 


\section{Tribology of a flexible spinal device: development of}

\section{experimental and numerical models}

Sophie Le Cann ${ }^{* 1,2}$, Julien Chaves-Jacob ${ }^{1}$, Jean-Marie Rossi ${ }^{1,2}$, Jean-Marc Linares ${ }^{1}$ and Patrick Chabrand $^{1,2}$

${ }^{1}$ Aix-Marseille Université, CNRS, ISM UMR 7287, 13288 Marseille cedex 09, France

${ }^{2}$ APHM, Hôpital Sainte-Marguerite, Institute for Locomotion, 13009, Marseille, France

* Corresponding author

Email : sophie.le-cann@univ-amu.fr 
Abstract: New fusionless devices are being developed to get over the limits of actual spinal surgical treatment, based on arthrodesis. However, due to their recentness, no standards exist to test and validate those devices, especially concerning the wear. A new tribological first approach to the definition of an in vitro wear protocol to study wear of flexible and sliding spinal devices is presented in this article, and was applied to a new concept. A simplified synthetic spine portion (polyethylene) was developed to reproduce a simple supra-physiological spinal flexion $\left(10^{\circ}\right.$ between two vertebrae). The device studied with this protocol was tested in wet environment until 1 million cycles (Mc). We obtained an encouraging estimated wear volume of same order of magnitude compared to similar devices. An associated finite element (FE) numerical model has permitted to access contact information and study the effect of misalignment of one screw. First results could point out how to improve the design and suggest that a vertical misalignment of a screw (under or over-screwing) has more impact than a horizontal one. 2016 Wiley Periodicals, Inc. J Biomed Mater Res Part B: Appl Biomater, 108B: 104-111, 2018.

Key Words: flexible sliding spinal device, tribology, wear, finite element analysis

How to cite this article: Le Cann S, Chaves-Jacob J, Rossi J-M, Linares J-M, Chabrand P. 2018. Tribology of flexible and sliding spinal implants: Development of experimental and numerical models. J Biomed Mater Res Part B 2018:106B:104-111.

\section{INTRODUCTION}

The most common surgical spinal treatments are based on arthrodesis, associated with metallic implants, ${ }^{1}$ correcting and fusing the pathologic spine. This current treatment is not suitable to preserve spine biomechanics, and could induce new pathologies such as adjacent segment degeneration. ${ }^{2}$ To overcome those limits, new "fusionless" implants are being developed. Some implants are composed of flexible rods ${ }^{3,4}$ aiming at better distributing the loads between the device and the spine and allowing some mobility to avoid fusion. Others combine metallic rods and sliding screws, ${ }^{5-8}$ allowing movement of the spine. However, these sliding systems present some issues such as the metallic wear debris released ${ }^{5,9}$ and the high stiffness of the devices that could lead to pullouts ${ }^{10}$ and spontaneous fusions. ${ }^{11,12}$ To overcome such issues, a new device has been designed, combining flexible rods and sliding screws. Composed of pedicle screws containing inside their head a holed rotating ball (ceramic), this implant is conceived to allow movement of the rod through rotations ( 3 degrees of freedom, dof] and translation (1 dof). Moreover, the device is made of flexible rods (PEEK) that aim to allow movement of the instrumented spine, preserving spinal biomechanics.
One main issue concerning sliding devices is their wear behavior. The existing spinal standards are adapted to fusion rods (such as ASTM F1717 13 or ISO $12189^{14}$ ) or total disc prostheses (such as ISO $18192^{15}$ or ASTM F2423 ${ }^{16}$ ) and cannot be used to study the wear behavior of sliding devices. To the knowledge of the authors, the work done by Singh et al. ${ }^{17}$ is the only one studying the in vitro wear of a sliding spinal system, composed of metallic rods, ${ }^{5}$ adapting an existing standard. ${ }^{14}$ Yet, this protocol imposed a limited flexion of $\pm 3^{\circ}$ to the rods, not adapted to test flexible devices.

A new experimental approach to study the wear behavior of new flexible and sliding spinal systems is presented in this article and was conceived to realize an overall sagittal flexion of $40^{\circ}$ over 5 vertebrae, corresponding to $10^{\circ}$ of flexion between two consecutive vertebrae. This set-up was applied to a new prototype of flexible and sliding system. The proposed protocol, conceived to be easy to set-up and reproducible, enables to test a portion of a flexible and sliding spinal device, subjecting it to a simplified spine kinematics. The experiment has been modeled in finite elements (FE) to access contact information inside the screws and study the effect of misalignment of the screws that could occur during implantation. 


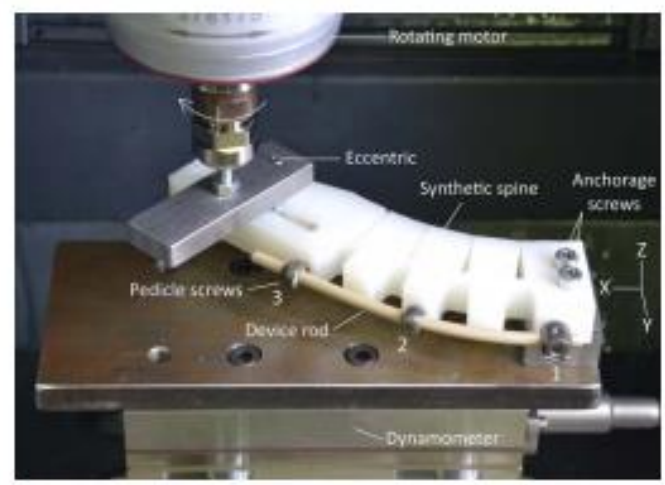

FGURE 1. Experimental set-up, with the tested prototype implanted

\section{MATERIALS AND METHODS}

Description of the experimental set-up

Synthetic spine kinematics. The experimental set-up is composed of a synthetic portion of spine made of polyethylene, with 5 blocks representing 5 lumbar vertebrae adapted from literature. ${ }^{17}$ The overall sagittal flexion is about $40^{\circ}$, approximately $10^{\circ}$ between two adjacent vertebrae, simulating a supra-physiological flexion of the spine. ${ }^{15,18}$ The synthetic spine was rigidly fixed, at one extremity, to a dynamometer through two anchorage screws (Figure 1). Flexion of the synthetic spine was achieved by an eccentric linked to a rotating motor controlled in speed $(120 \mathrm{rpm}$, corresponding to a rotation frequency of $2 \mathrm{~Hz}^{*}$ ). This set-up is designed to receive a flexible and sliding spinal device composed of pedicle screws and rods.

Application to a new flexible and sliding spinal device. This set-up was applied to a new prototype of flexible and sliding system. A portion composed of one locking non-deformable screw (screw 1 in Figure 1), two sliding ones (screws 2 and 3 in Figure 1) and a flexible rod was implanted. The center of the screw head was situated at $7.5 \pm 0.5 \mathrm{~mm}$ from the surface of the synthetic spine. Two adjacent screws were spaced of $50 \mathrm{~mm}$, corresponding to two vertebral levels. The device rod is $150 \mathrm{~mm}$ long with a diameter of $5.5 \mathrm{~mm}$. Wear tests were conducted in bovine serum environment (Dutscher SA) with a proteins concentration of $20 \mathrm{~g} / \mathrm{L}^{15}$ until 1 million cycles (Mc).

Measurements. A thermal camera (FLIR SC655) was used to validate the non-overheating of the tested device rod at the used rotation speed in dry condition, which was the most unfavorable condition. The maximum temperature was measured on the rod in contact with the second sliding screw (screw 3 in Figure 1), and remained below $50^{\circ} \mathrm{C}$, corresponding to one third of the fusion temperature of the tested material (Figure 2).

"Below the maximum of $5 \mathrm{~Hz}$ recommended by standards ${ }^{15}$

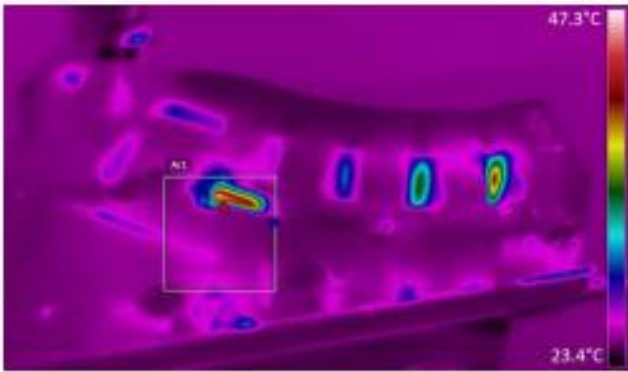

FIGURE 2. Thermal control during the wear tests

Anchorage forces were measured with a dynamometer (KISTLER ${ }^{2}$ type $9257 \mathrm{~B},-5$ to $5 \mathrm{kN}$ ) with and without the device, at a measurement frequency of $10 \mathrm{~Hz}$ over $10 \mathrm{flex}-$ ion cycles, reproduced 3 times. Those forces were used for validation of the finite element (FE) model, and device characterization: the difference of the maximum anchorage forces between the two configurations gives a value of the stiffness increase brought by the device.

Without disassembling the device from the synthetic spine (Figure 3), surface roughness measures were accurately characterized through optical measurements using the chromatic confocal sensing coordinate measuring machine (Micromesure2, STIL $L^{\circ}$, resolution about $40 \mathrm{~nm}$ in measurement direction). The critic linear wear profile was measured on the rod below the second sliding screw on a line of $25 \mathrm{~mm}$ with a 4- $\mu \mathrm{m}$ step, at different period stops. From these lines, the maximum wear depth evolution and the total sliding length were determined. Total wear volume was then extrapolated from the last critical linear wear profile (after $1 \mathrm{Mc}$ ) with a computer-aided design software reproducing the last passage of the ball sliding on the rod (Figure 4). The last profile was measured 6 times to evaluate the reproducibility of the measurement procedure, measuring the average and standard deviation of the maximum wear depth as well as the extrapolated wear volume. The lower/upper confidence bounds of these averages

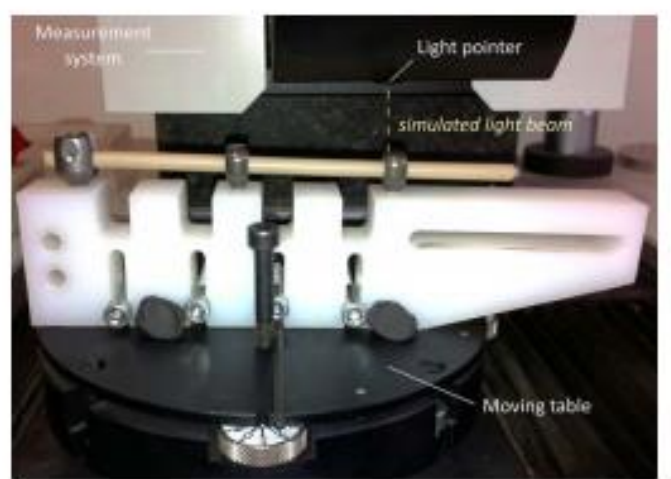

FGURE 3. 3D non-contact measurement system and the synthetic spine 


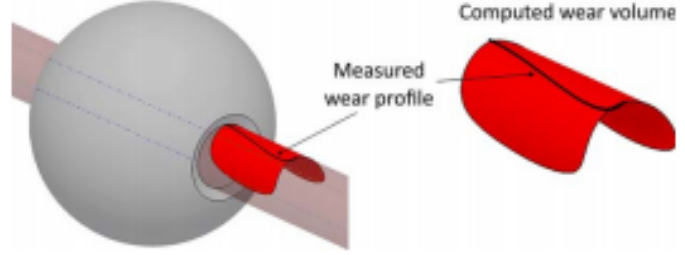

FGURE 4. Extrapolation of the wear volume from the measured critic linear wear profile

(maximum wear depth: $\pm 0.4 \mu \mathrm{m}$ and wear volume $\pm 0.02 \mathrm{~mm}^{3}$ ) were estimated with a confidence interval of $95 \%$

\section{Numerical modeling}

Definition of the finite element (FE) model. A threedimensional FE model of the experimental set-up was developed in Abaqus software (v6.11, SIMULIA ${ }^{\circ}$, see Figure 5). The model is composed of 3 main parts: the synthetic spine, the eccentric, and the tested device.

The movement of the synthetic spine was reproduced as realized in the experimental set-up. The eccentric part was modeled as a cylinder, assumed as a non-deformable rigid body piloted with a reference point ( $\mathrm{RPe}$, Figure $5 \mathrm{~A}$ ). This point was connected to another reference point representing the rotating center of the machine (RPr, Figure 5A) through a beam connector. Rotation of $360^{\circ}$ was applied to the RPr to realize one complete cycle.

The synthetic spine was meshed with 61,903 quadratic tetrahedral elements with a global element size of $5 \mathrm{~mm}$, refined at the folds and the groove. Mesh dependency was tested on a twice refined mesh. The eccentric was meshed with 3,509 quadratic tetrahedral elements.

The synthetic spine was assumed as linear elastic with a Young Modulus of $1.15 \mathrm{GPa}$ and a Poisson's ratio of 0.46 . The model was validated using anchorage forces measured experimentally. The Young Modulus of the synthetic spine was adjusted to fit the experimental anchorage forces recorded with and without the flexible rod of the device.

Self-contact of the synthetic spine during flexion as well as contact between the eccentric and the synthetic spine groove were modeled with hard normal contact and without tangential friction. The synthetic spine was clamped through its two anchoring screw holes to reproduce the experimental set-up.

The flexible rod of the tested device was modeled with 33,915 quadratic tetrahedral elements, as linear elastic with a Young Modulus of $3.6 \mathrm{GPa}$ and a Poisson's ratio of 0.4 (Ref. 19 and fabricant data). The locking screw (position 1 in Figure 5A) was modeled as a beam connector element, ${ }^{20}$ with all degrees of freedom blocked. The sliding screws (positions 2 and 3 in Figure 5A) were modeled with a combination of a 3D-modeled holed rotating ball and a connector to reproduce the behavior of the device (Figure 5B). For both sliding screws, the holed rotating ball, originally situated inside the sliding pedicle screws' head was assumed as a non-deformable rigid body. This rigid body was controlled by a reference point, located at its center (see RP in Figure SB), and set at a distance of $7.5 \mathrm{~mm}$ from the top of the vertebra, similar as the experimental implantation. This reference point was then connected the synthetic spine with a join and rotation connector, allowing 3 dof in rotation, enabling the holed ball to rotate around its reference point. Contact between the holed rotating ball and the rod was modeled with hard normal contact with a tangential friction coefficient determined through pin-on-disc friction test (see further section).

Measurement of the coefficient of friction of the sliding screw. In order to characterize the friction behavior of the device, friction tests were realized. Figure 6 presents the experimental protocol used to obtain the coefficient of friction (CoF) implemented in the finite element model, between the holed rotating ball and the flexible rod. In the presented experimental case, we used a rotating ball from the device as the pin (material 2, ceramic) and a standard dumbbell test piece for the disc (material 1, PEEK). A spring system was used to control the applied normal forces, to keep them as constant as possible and avoid rupture of the ceramic ball. Using the Hertz contact law, the normal force was fixed to $10 \mathrm{~N}$ to avoid plasticity of both materials. This applied force induces a contact pressure of $83 \mathrm{MPa}$ on the polymeric sample, remaining below the elastic limit of the material.

Friction tests were done in dry environment, with a displacement back and forth along a straight line of $80 \mathrm{~mm}$ (one cycle). We ran in the material with 1000 cycles of sliding at a speed of $100 \mathrm{~mm} / \mathrm{s}$, ensuring the non-overheating
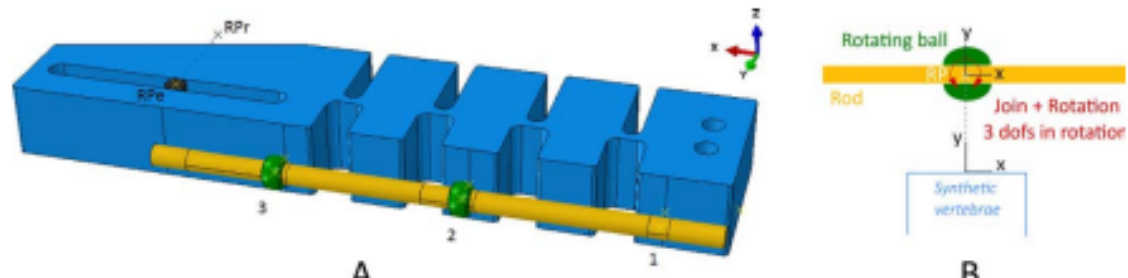

B

FGURE 5. 3D modeling of the experimental set-up. A: whole model, RPe represents the eccentric center, RPr the rotation center of the motor, and 1, 2 and 3 the 3 screws implanted and modeled with connectors. B: detail of the sliding screw model with the use of a connector element (sagittal view) 


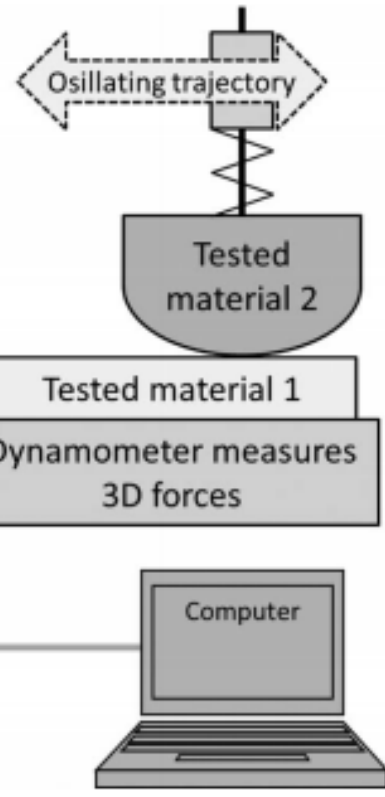

FIGURE 6. Scheme of the pin-on-disc test to determine the friction coefficient of the rod sliding inside the rotating ball of the device

of the samples by thermal control. Normal forces (Fn) and feed direction forces $(\mathrm{Ff})$ were then recorded during 5 cycles at a speed of $10 \mathrm{~mm} / \mathrm{s}$ with the same dynamometer at an acquisition frequency of $100 \mathrm{~Hz}$. At each measuring point the ratio $\mathrm{Ff} / \mathrm{Fn}$ was computed and averaged to obtain the coefficient of friction.

Tested configurations of the numerical model. Once the basic numerical model was created, it was modified to evaluate the impact of misalignment of one screw, the extreme one (second sliding screw), on the behavior of the device. To realize these configurations, the connector join and rotation, modeling the extreme screw, was changed to slot and rotation with a locking condition. The configurations done were:

- Vertical misalignment: the extreme screw was "underscrewed" or "over-screwed" from half or complete turn, i.e. $1.95 \mathrm{~mm}$ for this type of screw.

- Horizontal misalignment: the extreme screw was "implanted" not in the alignment of the two other screws but deviated from 2 and $3 \mathrm{~mm}$ laterally.

Measured parameters. From the numerical modeling, the maximum von Mises stress (MPa) of the rod and its localization were collected. The two sliding screws were also studied: the total sliding length ( $\mathrm{mm}$ ), the maximum of contact pressure on the rod induced by the rotating ball (MPa) and the maximum of the normal forces $(\mathrm{N})$ were collected.

\section{RESULTS}

Experimental study

Stiffness of the device. Figure 7 represents the planar anchorage forces ( $x$ - and $y$-directions are shown in Figure 1) obtained during the experimental measurements with and without the device. The implantation of the tested device increased the maximal anchorage forces respectively by $40 \%$ and $26 \%$ for the $x$ - and $y$-directions.

Wear tests. To reach 1 million cycles (Mc) at a frequency of $2 \mathrm{~Hz}$, including several stops to measure wear, the global experiment lasted around 8 days.

Maximum wear depths of the rod below the second sliding screw at different stop points of the lubricated tests are presented in Table I. The evolution of these depths is nonlinear; with a rapid increase before the 500,000 cycles, reaching a maximum wear depth of $13 \mu \mathrm{m}$ and a slower decrease after, reaching a maximum of $15.2 \pm 0.4 \mu \mathrm{m}$ at $1 \mathrm{Mc}$.

Figure 8 shows the critical linear profile on the rod induced by the second sliding screw after $1 \mathrm{Mc}$. The rod surface has been smoothed by the rotating ball during the

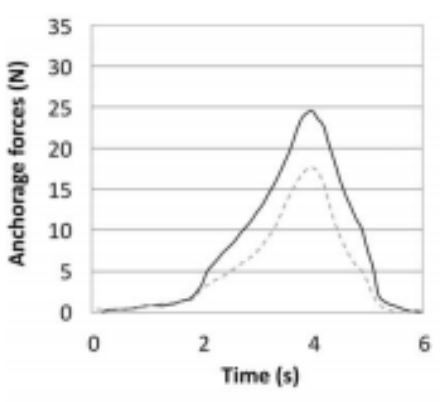

A

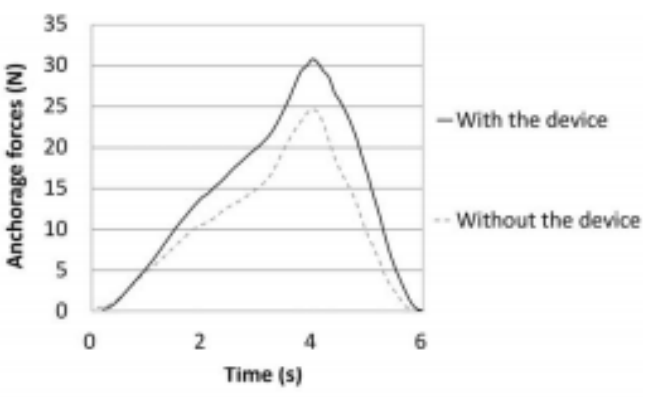

FGURE 7. Planar experimental anchorage forces for the $x$-direction (A) and the $y$-direction (B) with and without the tested device, during one flexion cycle 
TABLE I. Maximum Wear Depths of the Rod Under the Second Sliding Screw, at Different Stop Points During the Experimental Test

\begin{tabular}{lccccccccc}
\hline Cycles & 3000 & 10,000 & 30,000 & 100,000 & 150,000 & 300,000 & 500,000 & 850,000 & 1 million \\
\hline Maximum wear depth $(\mu \mathrm{m})$ & 1.5 & 2.5 & 3.5 & 5 & 6 & 9 & 13 & 15 & 15.2 \\
\hline
\end{tabular}

wear tests, compared to the non-worn zones. The sliding length is about $20 \mathrm{~mm}$ and the reference wear volume extrapolated is $0.43 \pm 0.02 \mathrm{~mm}^{3} / \mathrm{screw} / \mathrm{Mc}$.

Coefficient of friction of the sliding screw. The pin-on-disc tests realized between the ceramic and the PEEK gave an average friction coefficient of $0.11 \pm 0.01$, implemented in the numerical model.

\section{Simulation results}

Mesh dependency. The mesh dependency of the synthetic spine was tested (without the device) with a mesh twice refined; 130,000 elements (Mesh 2) compared to 61,903 of the used model (Mesh 1). Anchoring forces are presented in Figure 9; maximum forces differ at maximum by $1.1 \%$ and $1.3 \%$, respectively on $x$ - and $y$-axis.

Validation through experimental anchoring forces. Figure 10 shows the anchoring forces obtained numerically and experimentally for one flexion cycle of the synthetic spine alone (A), and with the device (B). The numerical curves (continuous lines) correctly fit the experimental ones (dotted lines). The addition of the tested device increased the maximal numerical anchorage forces by $34 \%$ in both the $x$ - and $y$-directions.

Numerical tests results. Results for the two sliding screws are presented in Table II, for the ideal configuration and the tested screw misalignments. Most maximal values coincide with the maximal flexion of the device.

The maximum stresses on the rod were always observed locally near the locking screw (screw 1 in Figure 5A), approximately at the time step of maximum flexion. Around $90 \mathrm{MPa}$ were measured at maximum for the ideal configuration, and slightly modified by the misaligned configurations (7\% at maximum).

The sliding lengths of the aligned configuration were of $10 \mathrm{~mm}$ for the central screw and $16 \mathrm{~mm}$ for the extreme

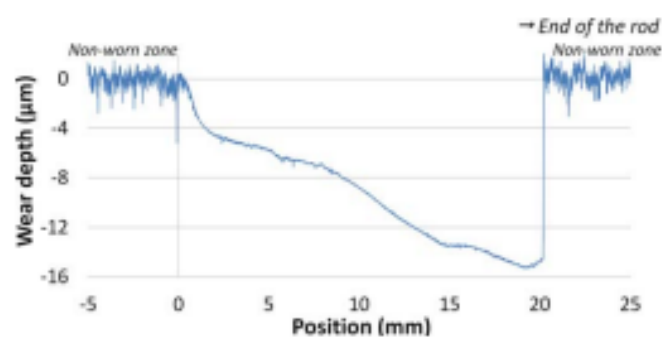

FGURE 8. Critical wear linear profile of the second sliding screw after 1 million cycles one, and were not affected by the misalignments of the extreme screw.

The normal forces are the forces on the $y$-axis of the local reference for each screw, as shown in Figure 5(B); a positive force means pullout, a negative one, compression. In the ideal configuration, the central screw was subjected to compressive forces of a maximum around $20 \mathrm{~N}$ and the extreme screw to pullout forces of the same maximum intensity. These forces were, respectively, reduced and increased by an under- and over-screwing of the extreme screw; at maximum by $35 \%$ for the central screw and $30 \%$ for the extreme screw for one turn displacement. Horizontal misalignments had no impact on those $y$-forces, however, they induced new forces on the screws, opposite to the direction of the misalignments ( $z$ direction, Figure 5B) of maximum $17 \mathrm{~N}$ for the central screw and $7 \mathrm{~N}$ for the extreme screw.

Figure 11 shows an example of the contact pressure on the rod, induced by the rotating balls, for the aligned configuration. For the central screw (screw 2 in Figure 5A), three contact zones are observed, two at the inferior surface of the rod and one at the superior surface. Only one superior contact zone is observed for the second sliding screw (screw 3 in Figure 5A). Maximum contact pressure was recorded at both sliding screws for all configurations (Table II). The under-screwing of the extreme screw reduced the contact pressure on both sliding screws ( $-19 \%$ at maximum) whereas the over-screwing had the opposite impact, in similar percentages.

\section{DISCUSSION}

Spinal fusionless devices have been developed for about 10 years ${ }^{5,21}$ with new wear behavior that needs to be characterized; however, actual spinal standards are not adapted. In this article, first experimental and numerical tribological approaches have been developed to investigate the wear of

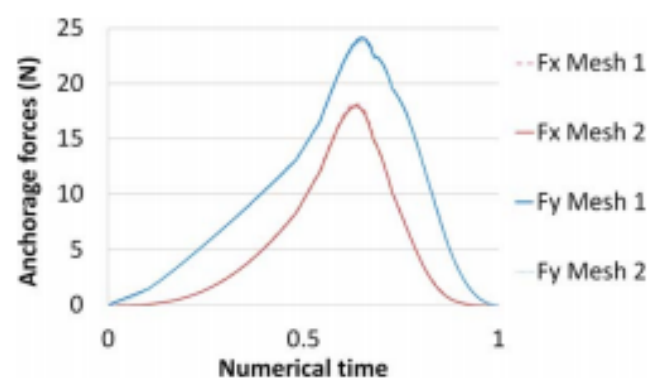

FGURE 9. Comparison of the anchorage forces for two different mesh sizes of the synthetic spine, without the device implanted, during one flexion cycle 


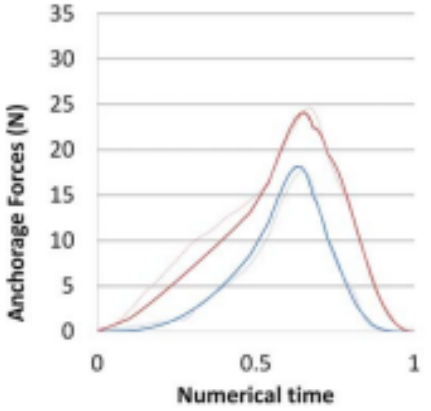

A

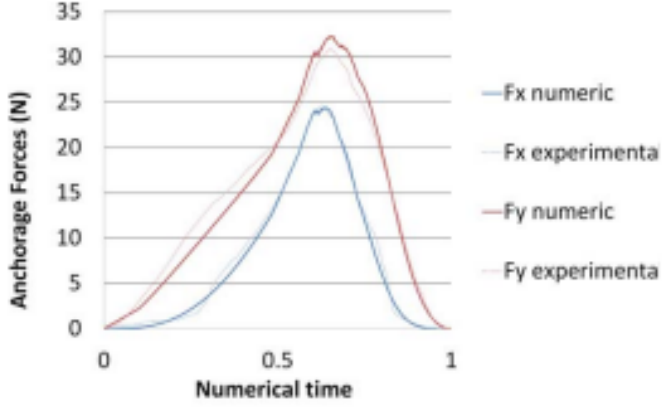

B

FGURE 10. Validation of the numerical model with the experimental anchorage forces, for one flexion cycle. A: synthetic spine alone and B: synthetic spine with the device

flexible sliding devices. The in vitro wear protocol designed here aspired to be simple and easy to reproduce, mimicking a spinal portion of 5 vertebrae inducing a critical supraphysiological flexion. A numerical model was associated, and enabled to evaluate the impact of screws misalignment, that can occur during surgery. A new prototype was studied through this protocol and gave encouraging results.

After 1 million cycles of flexion the extrapolated experimental wear volume was $0.43 \pm 0.02 \mathrm{~mm}^{3} / \mathrm{screw}$. This is encouraging result when compared to the literature, with a wear rate of the same order of magnitude compared to spinal systems suffering wear such as sliding rods and intervertebral disc prostheses ranging from 0.1 to $1.1 \mathrm{~mm}^{3} /$ component/Mc (Figure 12). Moreover, a recent study of a retrieval of spinal sliding device attested of $12.5 \pm 1.5 \mathrm{~mm}^{3}$ wear per year ${ }^{8}$; and concerning total hip prosthesis, widespread nowadays, it is not uncommon to find wear rates ranging from a few dozen to more than a hundred $\mathrm{mm}^{3}$ / $\mathrm{Mc}^{22}$ without leading to failure of the prostheses. Assuming implantation of a dozen screws, the predicted wear rate of a complete device would be around $5 \mathrm{~mm}^{3} / \mathrm{Mc}$, below the published values.

The evolution of the maximum wear depth on the rod along the tests is nonlinear (Table 1); with a rapid increase before half million cycles and then a slower decrease until 1 Mc. This suggests a running in phase at the beginning of the use of the device. Moreover, the rod has been smoothed by the sliding of the screw during the wear tests (Figure 8),

TABLE II. Numerical Results According to the Tested Configurations

\begin{tabular}{|c|c|c|c|c|c|c|c|}
\hline & Rod & First & ding screw & crew 2) & Second sl & ing screw I & ew 3) \\
\hline & $\begin{array}{c}\text { Max Von } \\
\text { Mises (MPa) }\end{array}$ & $\begin{array}{l}\text { Sliding } \\
\text { length } \\
\text { (mm) }\end{array}$ & $\begin{array}{c}\text { Max } \\
\text { Contact } \\
\text { Pressure } \\
\text { (MPa) }\end{array}$ & $\begin{array}{c}\text { Max } \\
\text { normal } \\
\text { forces (N) }\end{array}$ & $\begin{array}{c}\text { Sliding } \\
\text { lengths (mm) }\end{array}$ & $\begin{array}{c}\text { Max } \\
\text { Contact } \\
\text { Pressure } \\
\text { (MPa) }\end{array}$ & $\begin{array}{c}\text { Max } \\
\text { normal } \\
\text { forces (N) }\end{array}$ \\
\hline Ideal configuration & 93.5 & 9.76 & 6.24 & -21.3 & 16.1 & 1.39 & 20.4 \\
\hline Influence of vertical & Inment of the $\mathrm{e}$ & reme scr & & & & & \\
\hline Under-screwing & & & & & & & \\
\hline-1 turn & 100.4 & 9.63 & 5.06 & -13.9 & 16.0 & 1.21 & 14.4 \\
\hline & $7.4 \%$ & $-1.3 \%$ & $-19 \%$ & $-35 \%$ & $-0.6 \%$ & $-13 \%$ & $-29 \%$ \\
\hline$-1 / 2$ tum & 97 & 9.64 & 5.65 & -17.6 & 16.1 & 1.27 & 17.4 \\
\hline & $3.7 \%$ & $-1.2 \%$ & $-9.5 \%$ & $-18 \%$ & $0 \%$ & $-8.6 \%$ & $-15 \%$ \\
\hline Over-screwing & & & & & & & \\
\hline$+1 / 2$ turn & 90.2 & 9.71 & 6.82 & -25.0 & 16.1 & 1.50 & 23.6 \\
\hline & $-3.5 \%$ & $-0.5 \%$ & $9.3 \%$ & $17 \%$ & $0 \%$ & $7.98 \%$ & $16 \%$ \\
\hline+1 turn & 87.1 & 9.70 & 7.38 & -28.7 & 16.1 & 1.54 & 26.6 \\
\hline & $-6.8 \%$ & $-0.6 \%$ & $18 \%$ & $35 \%$ & $0 \%$ & $11 \%$ & $30 \%$ \\
\hline Influence of horizon & lignment of th & extreme : & & & & & \\
\hline$+2 \mathrm{~mm}$ & 94.4 & 9.68 & 6.24 & -21.3 & 16.1 & 1.40 & 20.5 \\
\hline & $1 \%$ & $-0.8 \%$ & $0 \%$ & $0 \%$ & $0 \%$ & $0.7 \%$ & $0 \%$ \\
\hline$+3 \mathrm{~mm}$ & 95.1 & 9.66 & 6.41 & -21.2 & 16.1 & 1.39 & 20.5 \\
\hline & $1.7 \%$ & $-1 \%$ & $2.7 \%$ & $-1 \%$ & $0 \%$ & $0 \%$ & $0 \%$ \\
\hline
\end{tabular}

1 turn corresponds to $1.95 \mathrm{~mm}$. The normal forces are the forces on the $y$ axis of the local reference as shown in Figure 5(B). 

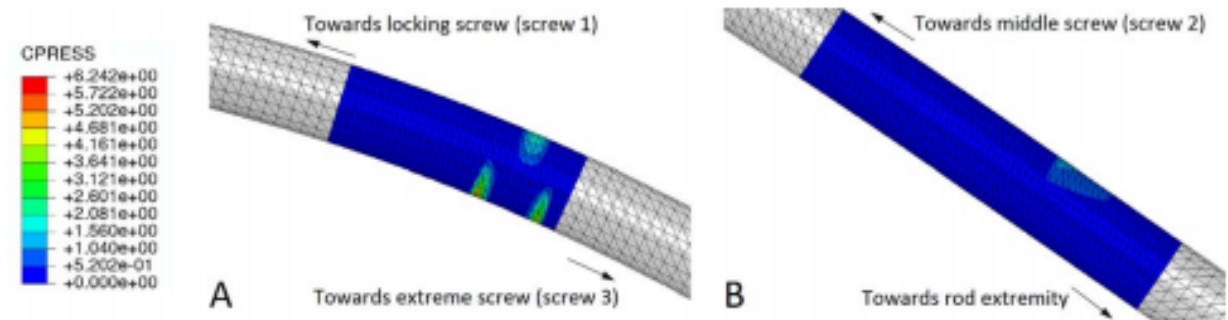

FIGURE 11. Sagittal view of the contact pressure zones induced on the rod by the rotating balls at A: the middle position (screw 2) and B: the extreme position (screw 3), at maximum flexion

compared to the non-worn zones. These results advise to well study the surface aspects at the sliding contact (here $\mathrm{rod} / \mathrm{rotating}$ ball), and work on the run in process of the device before implantation.

To build an associated FE model, the friction coefficient of the device had to be characterized. To the knowledge of the authors, the couple ceramic/PEEK is currently not used in implants and no value could be found in the literature. A friction coefficient of $0.11 \pm 0.01$ was measured, consistent with the value of 0.1 measured for the alumina/PEEK couple in dry rolling contact. ${ }^{23}$

The model was designed to be easy to set up, reproducing the kinematic behavior of the prototype with simplified connector elements, avoiding some contact issues and reducing calculation time. It was validated with anchorage forces, fitting the experimental ones (Figure 10) by adapting the Young Modulus of the synthetic spine. Moreover, considering the width of the rotating ball, the sliding length for the second screw obtained numerically in the aligned configuration fitted the experimental measurements.

The stiffness of the prototype was evaluated experimentally by the measure of the anchorage forces, and verified numerically. This can allow to test different configurations of implantation such as the screws positions and number as well as the rod diameter, to evaluate their impact on rigidity.

Through the numerical model, we could access maximum stresses on the rod, contact pressure at the rotating ball/rod interface and normal forces existing at the screws during a supra-physiological flexion, and evaluate the effect of misalignment of a screw.

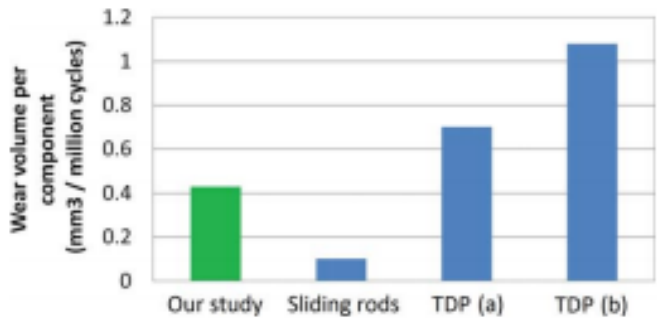

FGUUE 12. Comparison of the wear of the tested device with the literature. Per screw for our study and a sliding system (adapted from Ref. 17) or per intervertebral disc for Total Disc Prostheses (TDP) with PEEKUPEEK contact (a adapted from Ref. 25 and $b$ from Ref. 26)
For the aligned configuration, the maximum Von Mises stresses reached locally $93.5 \mathrm{MPa}$ on the rod, near the locking screw, and the misalignments configurations had few impact. The elastic limit of the rod material in tension is around $90 \mathrm{MPa}$ (fabricant data), so according to the numerical results, the limit is already reached with the aligned configuration. However, the flexion imposed here to the device is non-physiological and had been set to evaluate the device in a critical way.

As contact pressure is directly linked to wear by the sliding speed, it is a crucial parameter to evaluate. The contact pressure induced on the rod by the rotating balls was different between the two sliding screws (see Figure 11). Those pressure patterns were also observed experimentally, with a wear induced to the inferior surface of the rod for the central screw, and the superior rod surface for the extreme screw. Moreover, three localized contact zones were observed for the central screw, coming from the sharp geometry of the rotating ball, inducing high and localized pressures on the rod [Figure 11(A)]. This design does not seem efficient and should be corrected in the future. Only one superior contact zone was observed for the second sliding screw, consistent with the wear profile measured experimentally (Figure 8). For all configurations, the maximum of contact pressure was observed on the rod below the central screw. Under-screwing of the extreme screw diminished the contact pressure both at the central and extreme positions, what was expected as it is similar as a less flexed configuration for the device. Over-screwing and lateral misalignment both increased the contact pressures, the vertical misalignment being worse (Table II). The sliding lengths were very little impacted by the misalignments, suggesting that the wear may be rather affected by the contact pressure.

During the flexion, the central sliding screw is subjected to negative normal forces, meaning compression forces on the screw, explaining the localization of the contact zone at the inferior surface of the rod. In the opposite, the extreme screw is subjected to positive forces, meaning pullout forces, coherent with the contact zones, and expected because it is the end of the construct. The vertical misalignments of the extreme screw affected these forces (up to $30-35 \%$ ) whereas the horizontal ones had very little effect, this can be explained because it is a small displacement ( $\max 3 \mathrm{~mm}$ ) compared to the overall flexion of the device. The horizontal 
misplacement, however, induced new forces opposite to the misalignment direction [z-direction Figure $5(B)]$. In all cases, intensities of these forces remained below $30 \mathrm{~N}$, far from the pullout limits of such screws tested on porcine spines, around few hundreds of Newton. ${ }^{24}$

This protocol could not be validated on other devices or with in vivo retrieved implants because no flexible and sliding spinal devices are currently implanted; however, comparison values will be obtained in the future through anima experimentations. The polymeric material used for the synthetic spine may not represent completely the rigidity of a spine and may affect these results; this should be considered in the future. Additional experimental test campaigns will be done to confirm the first results obtained here, and quantify wear in misaligned configurations. The numerical model is promising and numbers of other parameters could be studied in the future, such as a new smooth design of the rotating ball, the influence of the rod diameter, the misalignment of the central screw, different rod materials, and so forth. Finally, those approaches could be spread to test a larger spinal portion as well as other flexible sliding spinal systems to be developed.

\section{CONCLUSION}

A first in vitro protocol was proposed to characterize the tribological behavior of new flexible sliding spinal systems. A numerical model reproducing the experiment was also developed to access contact information and test the influence of screw misalignment. This protocol has been tested on a prototype. A friction coefficient of 0.11 was measured for the couple ceramic/PEEK, necessary to model the device. We obtained first encouraging wear values and could detect improvements to realize such as the geometry of the implant.

\section{REFERENCES}

1. Cotrel $Y$, Dubousset J. A new technic for segmental spinal osteosynthesis using the posterior approsch. Rev Chir Orthop Reparatrice Appar Mot 1984;70:489-494.

2. Virk SS, Niedermeier S, Yu E, Khan SN. Adjacent segment disease. Orthopedics 2014;37:547-555.

3. Highsmith JM, Tumialan LM, Rodts Jr GE. Flexible rods and the case for dynamic stabilization. Neurosurg Focus 2007;22:E11.

4. Barrey CY, Ponnappan RK, Song J, Vaccaro AR. Biomechanical evaluation of pedicle screw-based dynamic stabilization devices for the lumbar spine: A systernatic review. SAS J 2008;2:159-170.

5. McCarthy RE, Sucato D, Turner JL, Zhang H, Henson MAW, McCarthy $\mathrm{K}$. Shilla growing rods in a caprine animal model: $A$ pilot study. Clin Orthopsed Relat Res 2009;468:705-710.

6. Ouellet J. Surgical technique: Modern Luqué Trolley, a self-growing rod technique. Clin Orthopaed Relat Res 2011;469:1358-1387.

7. Li K, Zhao S, Wei $X$, Wang $X$, Sun J, He Y, Zhen J. A preliminary study on a novel growth guidance rod system for early-onset scoliosis in a sheep model. Spine 2015;40:767-772.
8. Lukina E, Kollerov M, Meswania J, Wertheim D, Mason P. Wagstaff P, Laka A, Noordeen H, Yoon WW, Blunn G. Analysis of retrieved growth guidance sliding LSZ-4D devices for early onset sooliosis and investigation of the use of nitinol rods for this sys. tem. Spine 2015;40:17-24.

9. Lukina E, Laka A, Kollerov M, Sampiev M, Mason P, Wagstaff P, Noordeen H, Yoon WW, Blunn G. Metal concentrations in the blood and tissues after implantation of titanium growth guidance sliding instrumentation. Spine J 2016;16:380-398.

10. Yang JS, Sponseller PD, Thompson GH, Akbarnia BA, Emans JB, Yazici M, Skaggs DL, Shah SA, Salari P, Poe-Kochert C. Growing rod fractures: risk factors and opportunities for prevention. Spine 2011;36:1639-1644.

11. Cahill PJ, Marvil S, Cuddihy L, Schutt C, Idema J, Clements DH, Antonacci MD, Asghar J, Samdani AF, Betz RR. Autofusion in the immature spine treated with growing rods. Spine 2010;35:E1199.

12. Kim WJ, Lee SH, Shin SW, Rivard CH, Coillard C, Rhalmi C. The influence of fixation rigidity on intervertebral joints. An experimental comparison between a rigid and a flexible system. J Korean Neurosurg Soc 2005;37:364-369.

13. ASTM F1717. Standard test methods for spinal implant constructs in a vertebrectorny model. ASTM International 2004;1-18.

14. ISO 12189. Implants for surgery - Mechanical testing of implantable spinal devices - Fatigue test method for spinal implant assemblies using an anterior support. International Organization for Standardization 2008;1-11.

15. ISO 18192. Implants for surgery - Wear of total intervertebral spinal disc prostheses. International Organization for Standardization 2011;1-26.

16. ASTM F2423. Standard guide for functional, kinematic, and wear assessment of total disc prostheses. ASTM International. 2011.

17. Singh V, Simpson J, Rawlinson J, Hallab N. Growth guidance system for early-onset scoliosis: comparison of experimental and retrieval wear. Spine 2013;38:1546-1553.

18. Kapandji IA. The Physiology of the Joints: The Trunk and the Vertebral Column, Volume 3, 2e. 2 edition. Edinburgh: Churchill Livingstone; 1974, 256.

19. Kurtz SM, Devine JN. PEEK biomaterials in trauma, orthopedic, and spinal implants. Biomaterials 2007;28:4845-4869.

20. Rohlmann A, Zander T, Burra NK, Bergmann G. Flexible nonfusion scoliosis correction systems reduce intervertebral rotation less than rigid implants and allow growth of the spine: a finite element analysis of different features of orthobiom ${ }^{\mathrm{TM}}$. Eur Spine J 2007:17:217-223.

21. Sengupta DK, Webb JK, Mehdian H. Luque-Trolley growing-rod construct in the surgical treatment of early onset idiopathic scoliosis-a long term follow-up study. Spine J 2003;3:97-98.

22. Teoh SH, Chan WH, Thampuran R. An elasto-plastic finite element model for polyethylene wear in total hip arthroplasty. J Biomech 2002;35:323-330.

23. Sreenilayam-Raveendran R-K, Azarian MH, Morillo C, Pecht MG, Kida K, Santos EC, Honda T, Koike H. Comparative evaluation of metal and polymer ball bearings. Wear 2013;302:1499-1505.

24. Le Cann S, Cachon T, Viguier E, Miladi L, Odent T, Rossi J-M, Chabrand P. Pedicle screw fixation study in immature porcine spines to improve pullout resistance during animal testing. PLoS ONE 2015;10:e0127463.

25. Xin $\mathrm{H}$, Shepherd DET, Dearn KD. A tribological assessment of a PEEK based self-mating total cervical disc replacement. Wear 2013,303:473-479.

26. Grupp TM, Meisel HJ, Cotton JA, Schwiesau J, Fritz B, Blömer W, Jansson V. Alternative bearing materials for intervertebral disc arthroplasty. Biomaterials 2010,31:523-531. 